PROCEEDINGS OF THE

AMERICAN MATHEMATICAL SOCIETY

Volume 130, Number 12, Pages 3439-3445

S 0002-9939(02)06763-1

Article electronically published on July 2, 2002

\title{
TWISTED HOMOLOGY OF SYMMETRIC GROUPS
}

\author{
STANISLAW BETLEY \\ (Communicated by Ralph Cohen)
}

\begin{abstract}
We study the homology of symmetric groups $\Sigma_{n}$ with coefficients coming from the functor $T:$ finite pointed sets $\rightarrow A b$. We are primarily interested in the $\operatorname{limit} c o \lim _{n} H_{*}\left(\Sigma_{n} ; T([n])\right)$ where $[n]=\{0,1, \ldots, n\}$. Our main goal is to compare the described above situation with the case of general linear groups.
\end{abstract}

\section{INTRODUCTION}

Let $\Gamma$ be the category of finite pointed sets and let $T: \Gamma \rightarrow A b$ be a functor. The main purpose of the following note is to study the stable behaviour of the groups $H_{*}\left(\Sigma_{n} ; T([n])\right)$. Here the symbol $[n]$ denotes the finite set $\{0,1, \ldots, n\}$ and we are mainly interested in groups

$$
c o \lim _{n} H_{*}\left(\Sigma_{n} ; T([n])\right)
$$

which will be denoted $H_{*}(\Sigma ; T)$.

Let $\mathbf{R}$ denote the category of finitely generated free modules over a given ring $R$. Let $F: \mathbf{R} \rightarrow A b$ be a functor of finite degree. We proved in [B3] that in such a case

$$
H_{*}(G l(R) ; F)=0 .
$$

In the spirit of [BP-2] we can spell out this result by saying that stable K-theory groups for $F, K_{*}^{s}(R, F)$ are trivial for $0 \leq *$. In the following note we are going to define and calculate $K_{*}^{s}(\Sigma, T)$ for any functor $T \in A b^{\Gamma}$. We show that these groups are highly nontrivial even for functors of finite degree and the situation is completely different from the linear case. This is one of the reasons for writing this note. The calculations below show that the belief that $\Sigma_{n}$ is almost linear (over "the field with one element" see vdK p. 272]) has its limits.

This paper should be treated as a starting point for the study of the abelian category $A b^{\Gamma}$ and its homological algebra. The importance of this category comes, among others things, from the stable homotopy theory. If $X$. is a pointed simplicial set, then applying $T \in A b^{\Gamma}$ to it degreewise we obtain a simplicial abelian group whose stable (co)homotopy is expressable in terms of Ext and Tor groups in $A b^{\Gamma}$; compare [P2 Prop. 2.2]. This leads to many interesting computations; see [B4] and [BS].

Received by the editors October 4, 2000.

1991 Mathematics Subject Classification. Primary 20J06; Secondary 18G99.

The author was partially supported by the Polish Scientific Grant (KBN) 2 P03A 01113.

(C)2002 American Mathematical Society 
The paper is organized as follows. The first section contains basic observations and definitions. Among other things one can find there the definition of $K_{*}^{s}(\Sigma, T)$. In section 2 we prove that $K_{*}^{s}(\Sigma,$.$) are derived functors of the functor K_{0}^{s}(\Sigma,$.$) :$ $A b^{\Gamma} \rightarrow A b$. Section 3 contains the main calculations. In section 4 we find a nice interpretation of our results in terms of the category of surjections. We also prove there the stability result for $H_{*}\left(\Sigma_{n} ; T([n])\right)$. I believe that such a stability result has been well known for many years but I could not find it in the literature. Section 5 contains final remarks and comments.

\section{SECTION 1}

1.1. Cross-effects. Let $T$ be a functor from $A b^{\Gamma}$ such that $T([0])=0$. We now recall the definition of cross-effects of $T$ (for a careful discussion on this subject see [P1 section 2.3] or [P2]). Let $X \vee Y$ denote the sum of objects $X$ and $Y$ of $\Gamma$. The second cross-effect of $T$ is defined as the kernel of the obvious map $T(X \vee Y) \rightarrow T(X) \oplus T(Y)$. The construction is natural and we get in this way a bifunctor belonging to $A b^{\Gamma \times \Gamma}$. We will denote the value of it on the pair $X \times Y$ as $T(X \mid Y)$. In general the $n$th cross-effect of the functor $T$ is defined as the kernel of the map

$$
F\left(X_{1} \vee X_{2} \vee \ldots \vee X_{n}\right) \rightarrow F\left(X_{2} \vee \ldots \vee X_{n}\right) \times \ldots \times F\left(X_{1} \vee \ldots \vee X_{n-1}\right)
$$

It will be denoted $T\left(X_{1}|\ldots| X_{n}\right)$ and it is obvious that this way we get a functor from the $n$-fold product of $\Gamma$ to $A b$. Let $T_{k}$ denote $T([1]|\ldots|[1])$. Observe that $T_{k}$ comes with the natural action of $\Sigma_{k}$ coming from the permutation of summands. We have the following formula (compare [P1, formula 2.3.2]):

$$
T([n])=\bigoplus_{\gamma} T_{k}
$$

where the sum is taken over all pointed and order preserving injections $\gamma:[k] \rightarrow[n]$. We say that our functor is of degree $d$ if $T_{d+1}=0$ and $T_{d}$ is not trivial. As usual, the first assumption implies that $T_{s}=0$ for $s>d$. If our functor does not satisfy $T([0])=0$, then while speaking of its cross-effects we always have in mind the cross-effects of the kernel of the obvious map $T \rightarrow T([0])$.

1.2. Stable K-theory. Now let $T$ be a functor $T: \Gamma \rightarrow A b$. Let $F$ denote the homotopy fiber of the plus-construction $B \Sigma \rightarrow B \Sigma^{+}$where $\Sigma=\operatorname{colim} \Sigma_{k}$. We define

$$
K_{i}^{s}(\Sigma, T)=H_{i}(F ; \operatorname{colim} T([n]))
$$

where $\pi_{1}(F)$ acts on $\operatorname{colim} T([n])$ via the homomorphism $\pi_{1}(F) \rightarrow \Sigma$.

\subsection{Theorem.}

$$
H_{n}(\Sigma ; T)=\bigoplus_{p+q=n} H_{p}\left(\Sigma ; K_{q}^{s}(\Sigma, T)\right)
$$

where the action of $\Sigma$ on $K_{i}^{s}(\Sigma, T)$ is trivial.

We are not going to write the proof of this theorem. It is completely the same as the proof of Theorem 2 from [BP-2]. One will have to use Theorem 2.1 below instead of section 1 of [BP-2]. We leave the details for the interested reader. Observe only that similar to the linear case the groups $K_{*}^{s}(\Sigma, T)$ give us the trivial coefficients for calculating homology of the symmetric group with coefficients twisted by a functor. 
Obviously the interpretation that the groups $K_{*}^{s}(\Sigma, T)$ are obstructions for the projection map colim $T([n]) \rightarrow(\operatorname{colim} T([n]))_{\Sigma}$ to induce homology isomorphism $H_{*}(\Sigma, T) \rightarrow H_{*}\left(\Sigma, \operatorname{colim} T([n])_{\Sigma}\right)$ is also valid.

\section{SECTION 2}

We will show here that higher stable K-groups are derived functors of $K_{0}^{s}(\Sigma,$.$) .$ We do not claim any originality here - the proof of this fact is very similar to the one given in the linear case in [BP-2]. Let us formulate the theorem.

2.1. Theorem. The functors $K_{i}^{s}(\Sigma,):. A b^{\Gamma} \rightarrow A b$ are derived functors of $K_{0}^{s}(\Sigma,$.

Proof. The fact that a short exact sequence of functors from $A b^{\Gamma}$ induces a long exact sequence of stable K-theory groups is a trivial consequence of the definitions. The only thing which requires proving is the fact that $K_{*}^{S}(\Sigma, P)=0$ for $*>0$ if $P$ is a projective object in $A b^{\Gamma}$. Observe that the functors $\Gamma^{p}=Z \operatorname{Hom}_{\Gamma}([p],$.$) form a set$ of projective generators for $A b^{\Gamma}$. Moreover $\Gamma^{p}([n])=Z\left[n^{p}\right]=(Z[[n]-[0]])^{\otimes p} \oplus Z[0]$ as $\Sigma_{n}$-modules. Hence the proof follows easily from Lemma 2.2 below.

Let $t: \Gamma \rightarrow A b$ be defined via the formula $t(X)=(Z[X] \otimes C) / Z[0] \otimes C$ where $C$ is any abelian group. In $\left[\mathrm{K}\right.$, Chapter 4] Kassel proved that $H_{*}(\Sigma ; t)=$ $H_{*}\left(\Sigma ; K_{0}^{s}(\Sigma, t)\right)$. In other words using Theorem 1.3 we can say that $K_{i}^{s}(t)=0$ for $1 \leq i$. Below we prove a generalization of Kassel's observation to higher tensor powers of $t$.

2.2. Lemma. Let $t$ be as above with $C=Z$. Let $t^{k}$ denote the $k$-fold tensor product of $t$. Then $K_{i}^{s}\left(\Sigma, t^{k}\right)=0$ for $1 \leq i$.

Proof. In our considerations, while talking about $\Sigma_{n}$ we shall always assume that $k<n$. This is acceptable because we are interested only in arbitrarily large $n$ 's. Observe that $t[n]$ is a permutative $\Sigma_{n}$-module. The same is true for $t^{k}$ because of the formula $Z[X] \otimes Z[X]=Z[X \times X]$ for any finite set $X$. Hence $t^{k}([n])$ splits into the sum of induced $\Sigma_{n}$-modules over the set $O_{n}$ of orbits of the action of $\Sigma_{n}$ on $([n]-[0])^{k}$ and all of the summands are induced from $Z$ being a trivial module over the corresponding isotropy subgroup. Observe that for $k<n, O_{n}$ is independent of $n$. The reason for this is simple. Let $n>k$ and assume that in the sequence $\left(i_{1}, \ldots, i_{k}\right)$ of elements from $[n]-[0]$ we have $i_{j_{1}}, \ldots, i_{j_{s}} \leq k$ for some $s \leq k$. Then there is an element in $\Sigma_{n}$ which fixes $i_{j_{1}}, \ldots, i_{j_{s}}$ and sends $\left\{i_{1}, \ldots, i_{k}\right\}-\left\{i_{j_{1}}, \ldots, i_{j_{s}}\right\}$ to elements not exceeding $k$.

We shall denote $O_{n}$ as $O$ when $n$ is large. It is obvious that $K_{0}^{s}\left(\Sigma, t^{k}\right)$ is isomorphic to $\bigoplus_{O} Z$. Observe that the isotropy subgroup of an element $\left(i_{1}, \ldots, i_{k}\right) \in$ $([n]-[0])^{k}$ is isomorphic to $\Sigma_{n-f(k)}$ where $f(k)$ is the cardinality of the set $\left\{i_{1}, \ldots, 1_{k}\right\}$. We know by Shapiro's lemma that

$$
H_{*}\left(\Sigma_{n}, Z \otimes_{Z \Sigma_{n-f(k)}} Z \Sigma_{n}\right)=H_{*}\left(\Sigma_{n-f(k)} ; Z\right) .
$$

On the other hand we know by $[\mathbb{N}$ that homology of symmetric groups have good stability properties with trivial coefficients. Hence it is enough to observe that we have the following commutative diagram of homology groups:

$$
\begin{array}{ccc}
H_{*}\left(\Sigma_{n} ; Z \otimes_{Z \Sigma_{n-f(k)}} Z \Sigma_{n}\right) & \rightarrow & H_{*}\left(\Sigma_{n-f(k)} ; Z\right) \\
\downarrow & & \downarrow \\
H_{*}\left(\Sigma_{n+1} ; Z \otimes_{Z \Sigma_{n+1-f(k)}} Z \Sigma_{n+1}\right) & \rightarrow & H_{*}\left(\Sigma_{n+1-f(k)} ; Z\right)
\end{array}
$$


where vertically we have maps coming from the standard inclusion $[n] \rightarrow[n+1]$ and horizontal arrows are given by Shapiro's lemma. From this we know immediately that

$$
H_{i}\left(\Sigma ; t^{k}\right)=H_{i}\left(\Sigma ; \bigoplus_{O} Z\right)=H_{i}\left(\Sigma ; K_{0}^{s}\left(t^{k}\right)\right) .
$$

2.3. Remark. In Lemma 2.2 we could have an arbitrary abelian group $C$ instead of $\mathrm{Z}$. If this is the case, then $K_{0}^{s}=\bigoplus_{O} C^{\otimes k}$ and the proof is the same.

\section{SECTION 3}

This section is devoted to calculations of $K_{*}^{s}(\Sigma, T)$ for arbitrary functors. The main tool in dealing with them comes from (1.1.1). We start with the following lemma:

3.1. Lemma. Assume that $T \in A b^{\Gamma}$ and $T([0])=0$. Then $T([n])$ splits as a $\Sigma_{n}$-module

$$
T([n])=\bigoplus_{k=1}^{\infty} T_{n}^{k}
$$

where for given $k$

$$
T_{n}^{k}=\bigoplus_{\gamma} T_{k}
$$

and the latter sum is taken over all order preserving injections $\gamma:[k] \rightarrow[n]$.

Proof. The splitting as above certainly exists as a splitting of abelian groups by (1.1.1). We have to analyze the action of $\Sigma_{n}$ on $\bigoplus T_{k}$ for given $k$. The action of $\sigma \in \Sigma_{n}$ on $[n]=[1] \vee \ldots \vee[1]$ permutes the summands. Let $\gamma$ be an order preserving injection $[k] \rightarrow[n]$. If $\sigma \gamma$ is again order preserving, then the action of $\sigma$ on $T^{k}$ sends $T_{k}$ corresponding to $\gamma\left(\right.$ call it $T_{k}(\gamma)$ ) identically to $T_{k}(\sigma \gamma)$. In general, if $\gamma$ is as above, then there is precisely one $\alpha \in \Sigma_{k}$ such that $\sigma \gamma \alpha$ is an order preserving injection. Hence the action of $\sigma$ on $T_{n}^{k}$ identifies $T_{k}(\gamma)$ with $T_{k}(\sigma \gamma \alpha)$ by using the action of $\alpha^{-1}$ on $T_{k}$. Remember that $T_{k}$ comes equipped with the action of $\Sigma_{k}$ as was explained in subsection 1.1.

\subsection{Theorem.}

$$
K_{i}^{s}(\Sigma, T)=\bigoplus_{k=1}^{\infty} H_{i}\left(\Sigma_{k} ; T_{k}\right)
$$

Proof. This theorem is an easy consequence of the previous lemma. Observe first that the proof of Lemma 3.1 gives us the following formula for $T_{n}^{k}$ :

$$
T_{n}^{k}=T_{k} \otimes_{Z\left[\Sigma_{k} \times \Sigma_{n-k}\right]} Z\left[\Sigma_{n}\right]
$$

where $Z\left[\Sigma_{n-k}\right]$ acts trivially on $T_{k}$. Hence again by Shapiro's lemma and the Künneth formula

$$
H_{i}\left(\Sigma_{n} ; T_{n}^{k}\right)=H_{i}\left(\Sigma_{k} \times \Sigma_{n-k} ; T_{k}\right)=\bigoplus_{p+q=i} H_{p}\left(\Sigma_{n-k} ; H_{q}\left(\Sigma_{k} ; T_{k}\right)\right)
$$


where $\Sigma_{n-k}$ acts trivially on $\left.H_{q}\left(\Sigma_{k} ; T_{k}\right)\right)$. Going from $n$ to $n+1$ we just add one to $n-k$ in the formula above. We get

$$
H_{i}(\Sigma ; T)=\operatorname{colim}_{i}\left(\Sigma_{n} ; \bigoplus_{k} T_{n}^{k}\right)=\operatorname{colim} \bigoplus_{p+q=i} H_{p}\left(\Sigma_{n-k} ; \bigoplus_{k} H_{q}\left(\Sigma_{k} ; T_{k}\right)\right) .
$$

This implies our statement by an obvious inductive argument.

3.3. Remark. Observe that our result agrees with Lemma 2.2. It is easy to check that $\left(t^{p}\right)_{k}$ is a free $\Sigma_{k}$-module and hence $H_{*}\left(\Sigma_{k} ;\left(t^{p}\right)_{k}\right)=0$ for $*>0$.

\section{SECTION 4}

Let $\Omega$ be the small category of finite nonempty sets and surjections. We will assume that a typical object of $\Omega$ is $\langle n\rangle=\{1, \ldots, n\}$. We have a natural functor $\Omega \rightarrow \Gamma$ which sends $\langle n\rangle$ to $[n]$ and extends a surjection $f:\langle n\rangle \rightarrow\langle k\rangle$ to $\bar{f}:[n] \rightarrow[k]$ by the condition $\bar{f}(0)=0$. In [P1] Pirashvili proved the remarkable theorem ([P1], Theorem 3.1):

4.1. Theorem. There is a functor $c r: A b^{\Gamma} \rightarrow A b^{\Omega}$ which is an equivalence of categories.

The description of $c r$ is simple. If $T \in A b^{\Gamma}$, then $\operatorname{cr}(T)(\langle n\rangle)=T_{n}$. It is easy to see that if $f:\langle n\rangle \rightarrow\langle k\rangle$ is a surjection, then it sends the kernel of the corresponding map defining $T_{n}$ to $T_{k}$ (see (1.1.0)). Observe that if $T \in A b^{\Gamma}$ is of finite degree $d$, then the corresponding element of $A b^{\Omega}$ is given as a finite sequence $T_{1}, T_{2}, \ldots, T_{d}$ of $\Sigma_{1}, \Sigma_{2}, \ldots, \Sigma_{d}$-modules with additional structure. We can rewrite the considerations from section 3 as:

4.2. Theorem. Let $T \in A b^{\Gamma}$. Then the graded groups $H_{*}\left(\Sigma_{i} ; \operatorname{cr}(T)(\langle i\rangle)\right)$ for $i=$ $1, \ldots$ form the full sequence of trivial coefficients for calculating $H_{*}(\Sigma ; T)$.

In [N] Nakaoka proved that $H_{i}\left(\Sigma_{n-1} ; G\right) \rightarrow H_{i}\left(\Sigma_{n} ; G\right)$ is an isomorphism provided $i<n / 2$ and symmetric groups act trivially on the group $G$. A similar result holds for $H_{*}(G l(R) ; F)$ where $R$ and $F$ are as in the Introduction. We have:

4.3. Theorem. For any $T \in A b^{\Gamma}$ of finite degree $d$ the natural map

$$
\text { stab }: H_{i}\left(\Sigma_{n-1} ; T([n-1])\right) \rightarrow H_{i}\left(\Sigma_{n} ; T([n])\right)
$$

is an isomorphism provided $2 i+d<n$.

Proof. This theorem is an easy consequence of Theorems 1.3 and 3.2. Let us recall the formula for the interesting homology:

$$
\begin{aligned}
H_{i}\left(\Sigma_{n} ; T[n]\right) & =H_{i}\left(\Sigma_{n} ; \bigoplus T_{n}^{k}\right)=\bigoplus_{k=1}^{d} H_{i}\left(\Sigma_{n} ; T_{n}^{k}\right) \\
& =\bigoplus_{k=1}^{d} H_{i}\left(\Sigma_{k} \times \Sigma_{n-k} ; T_{k}\right)=\bigoplus_{k=1}^{d}\left(\bigoplus_{j+m=i} H_{j}\left(\Sigma_{n-k} ; H_{m}\left(\Sigma_{k} ; T_{k}\right)\right) .\right.
\end{aligned}
$$

Assume $d<n-1$. We can write the same formula as above for $\Sigma_{n-1}$. Then the map stab $: H_{i}\left(\Sigma_{n-1} ; T[n-1]\right) \rightarrow H_{i}\left(\Sigma_{n} ; T[n]\right)$ is induced by the standard embedding $\Sigma_{n-k-1} \rightarrow \Sigma_{n-k}$ and the identity on $H_{m}\left(\Sigma_{k} ; T_{k}\right)$. Hence we have reduced our twisted stability question to the same problem but with trivial coefficients, and 
now we can use Nakaoka's result. In order to have $s t a b$ be an isomorphism we must have $i<(n-d) / 2$ or equivalently $2 i+d<n$.

\section{SECTION 5}

We could have written this note in a slightly more general form. Namely, instead of talking about functors we could have used a more general category of bifunctors $T: \Gamma^{o p} \times \Gamma \rightarrow A b$ and obtained similar results as those in Theorems 1.3, 2.1, 3.2 and 4.3 while dealing with the conjugation action of $\Sigma_{n}$ on $T([n],[n])$. There is a conjecture that in the linear case the groups $K_{*}^{s}(T)$ are the same as $H_{*}(\mathbf{R}, T)$. These latter groups denote the homology groups of the category $\mathbf{R}$ with coefficients in a bifunctor $T$ and are often computable (see [JP], [B1], [FFSS]). The conjecture is proved in certain cases and this justifies the usefulness of theorems like 1.3. The full version of the conjecture is proved in $[\underline{\mathrm{S}}]$ and should be published soon. The conjecture is certainly true when we talk about functors treated as a special kind of bifunctors. But observe that this is not the case for the category $\Gamma$ because by general homological algebra methods we have (compare [BP-2, Section 3]):

5.1. Remark. If $T: \Gamma \rightarrow A b$, then the groups $H_{*}(\Gamma ; T)$ are trivial in positive dimensions and $H_{0}(\Gamma ; T)=T(0)$.

Now we see that the situation considered in this note really differs from the linear case. The case of functors is very transparent and that was the reason for writing the paper only for them. The homology of categories $\Gamma$ and $\mathbf{R}$ with functorial coefficients behave similarly but homological behaviour of the groups $\Sigma$ and $G l(R)$ is completely different.

\section{REFERENCES}

[B1] S. Betley. Calculations in THH-theory. J. Algebra 180 (1996), 445-458. MR 97a:19002

[B2] S. Betley. On stable K-theory with twisted coefficients. Contemporary Math. 199 (1996) 19-29. MR 98a:19002

[B3] S. Betley. Homology of Gl(R) with coefficients in a functor of finite degree. J. Algebra 150 (1992) 73-86. MR 93m:20069

[B4] S. Betley. Stable derived functors, the Steenrod algebra and homological algebra in the category of functors. Fundamenta Math. 168 (2001) 279-293. MR 2002f:18025

[BP-1] S. Betley and T. Pirashvili. Twisted (co)homological stability for monoids of endomorphisms. Math. Ann. 295 (1993), 709-720. MR 94g:18006

[BP-2] S. Betley and T. Pirashvili. Stable $K$-theory as a derived functor. J. of Pure and Appl. Alg. 96 (1994) 245-258. MR 96a:19002

[BS] S. Betley and J. Słomińska. New approach to the groups $H_{*}\left(\Sigma_{n}, L i e_{n}\right)$ by the homology theory of the category of functors. J. Pure and App. Alg. 161 (2001) 31-43. MR 2002e: 55020

[FFSS] V. Franjou, E.M. Friedlander, A. Scorichenko and A. Suslin. General linear and functor cohomology over finite fields. Ann. of Math. 150 (1999) 663-728. MR 2001b:14076

[JP] M. Jibladze and T. Pirashvili. Cohomology of algebraic theories. J. Algebra 137 (1991) 253-296. MR 92f:18005

[vdK] W. van der Kallen. Homology stability for linear groups. Inventiones Math. 60 (1980) 269-295. MR 82c:18011

[K] C. Kassel. Stabilisation de la K-theorie algebrique des espaces topologiques. Ann. Scient. Ec. Norm. Sup. 16 (1983) 123-149. MR 85j:18010

[N] M. Nakaoka. Decomposition theorem for homology groups of symmetric groups. Ann. of Math. 71 (1960) 16-42. MR 22:2989

[P1] T. Pirashvili. Dold-Kan type theorem for Г-groups. Math. Ann. 318 (2000) 277-298. MR 2001i:20112 
[P2] T. Pirashvili. Hodge decomposition for higher order Hochschild homology. Ann. Scient. Ec. Norm. Sup. 33 (2000) 151-179. MR 2001e:19006

[S] A. Scorichenko. Stable K-theory and functor homology over a ring. Preprint IAS, 2000.

Instytut Matematyki, University of Warsaw, ul. Banacha 2, 02-097 Warsaw, Poland

E-mail address: betley@mimuw.edu.pl 